\section{Phase Dependent Nonlinear Parametrically Excited Systems}

\author{
Bahareh Zaghari, Emiliano Rustighi, Maryam Ghandchi Tehrani
}

\begin{abstract}
Nonlinear Parametrically Excited (NPE) systems govern the dynamics of many engineering applications, from cablestayed bridges where vibrations need to be suppressed, to energy harvesters, transducers and acoustic amplifiers where vibrations need to be amplified. This work investigates the effect of different system parameters on the dynamics of a prototype NPE system. The NPE system in this work is a cantilever beam with an electromagnetic subsystem excited at its base. This system allows cubic stiffness, parametric stiffness, cubic parametric stiffness, and the phase difference between different sources of excitation to be varied independently to achieve different dynamic behaviours. A mathematical model is also derived, which provides theoretical understanding of the effects of these parameters, and allows the analysis to be extended to other applications.
\end{abstract}

\title{
Keywords
}

Nonlinear parametrically excited, base excitation, relative phase

\section{Introduction}

Dynamical systems are under Parametric Excitation (PE) when the system parameters vary periodically with time. In this case, the system can experience Parametric Resonance (PR), and the amplitude of the oscillation will be large (Nayfeh and Mook (2008)). Examples of parametrically excited systems subject to base excitation include cables moving due to the vibration of the deck in cable-stayed bridges. These systems also exhibit stiffness and inertia nonlinearities due to large deflections, which lead to Nonlinear Parametrically Excited (NPE) systems. Failures of engineering structures due to high vibrations have been caused by PR despite the implementation of damping systems. However, PR can also be used beneficially in some engineering systems. In microelectrical resonators, $\mathrm{PE}$ is exploited to perform electrical filtering or sensing without considerably amplifying noise (DeMartini et al. (2005); Hu et al. (2011)). PE is also used in the design of vibration energy harvesters to maximise the motion of the harvester at parametric resonance (Zaghari et al. (2014)). A vertically standing cantilever beam with a piezoelectric or an electromagnetic energy harvester under parametric excitation can produce significant energy if it overcomes an initial threshold amplitude. The initial threshold in PE systems is a function of linear damping in the system. When the level of input excitation exceeds the initial threshold, the amplitude of the response is not limited by the total linear damping present in the system, unlike in non-PE systems.

PE oscillators have been used to amplify or suppress the response amplitude (Chen et al. (2009)). The dynamic behavior of parametrically excited systems can be controlled to achieve periodic responses. Tusset et al. (2017) applied active control by employing nonlinear saturation control and a passive rotational magnetorheological damper to minimize the chaotic behavior of a parametrically excited pendulum. Zaghari et al. (2016) presented a parametric study to compare the gain associated with a base excited Linear PE (LPE) and a NPE system at several phase differences between the base excitation and the parametric excitation. In this study the optimum gain was found when the system parameters including parametric stiffness, positive cubic stiffness and positive/negative cubic parametric stiffness were considered. It was shown that the NPE system with a negative parametric stiffness has maximum gain when the phase difference between the base excitation and the parametric excitation is $\frac{\pi}{2}$.

Rhoads et al. (2008) demonstrated the potential of LPE in a macro-scale mechanical amplifier. They used a cantilever beam under longitudinal and transverse base excitation as an example of a parametrically excited system. They found $P E$ can increase the gain, where the gain is defined as the ratio between the amplitude of response without and with $\mathrm{PE}$, by changing the phase difference between the direct and parametric excitation. They identified maximum and minimum gain when the phase difference is near $\frac{3 \pi}{4}$ and $\frac{\pi}{4}$, respectively. The parametrically excited system presented by Rhoads et al. (2008) was considered for the cantilever beam excited at twice its natural frequency. Only the parametric stiffness was varied in the experimental model, and the effects of geometric or inertial nonlinearities in the system were not investigated, and their effect on the gain was not identified.

Institute of Sound and Vibration Research, University of Southampton, SO17 1BJ, UK

\section{Corresponding author:}

Bahareh Zaghari

Email: bahareh.zaghari@soton.ac.uk 
Rhoads and Shaw (2010) analysed the effect of phase difference on the gain when a nonlinear parameter, cubic stiffness due to deflection, inertia, or the transduction was considered in the model of a parametrically excited system. They concluded that the effect of phase difference in this NPE system is similar to the LPE system when the system is parametrically excited with an amplitude less than the linear instability threshold. However, this study was not conducted for parametric excitation with amplitude greater than the instability threshold. Kumar et al. (2011) demonstrated the phase dependence of a NPE oscillator for response amplification and suppression purposes. Their experimental work only studied the response amplitude at a fixed parametric frequency. The effect of the phase difference on the response amplitude of NPE systems has not been comprehensively investigated in the literature; this effect can impact the design of parametric amplifiers, vibration absorbers, and other applications where NPE systems are prevalent.

This paper investigates the effect of varying phase difference and excitation frequencies on the response amplitude of a NPE system with nonlinear terms, including cubic stiffness, cubic parametric stiffness, and nonlinear damping. The effect of phase difference on the upper and lower stable branches are presented. These branches determine the response amplitude of the nonlinear system, as well as the jumps between different branches of solutions. By using the results from these investigations, the parameters of NPE systems can be tuned to optimise the response amplitude for a given application. To model a tunable NPE system, a cantilever beam with an electromagnetic subsystem is used. The phase, cubic stiffness, cubic parametric stiffness, and nonlinear damping of the NPE system can be changed by altering the current of the electromagnetic system, which is more consistent and reproducible than altering properties of the equivalent NPE mechanical system. A mathematical model of this electromagnetic system is also derived with the method of averaging.

\section{Modelling}

To define a general NPE system with time-varying and nonlinear stiffness, a cantilever beam with an electromagnetic system is proposed. There are advantages of using an electromagnetic system over a cantileverbeam system subject to longitudinal and transverse base excitations. These include the facts that: the electromagnetic system excites the beam with a non-contact force, the base and parametric excitations can be applied independently in the electromagnetic case, and that the frequency and amplitude of the excitation force can be controlled more accurately using a DC/AC power source. Furthermore, the cubic and cubic parametric stiffness nonlinearities can be controlled independently in the electromagnetic system, which allows the effects of these nonlinearities on parametric amplification to be investigated more easily.

A clamped-free cantilever beam with an electromagnetic sub-system is fixed to a shaker plate (Figure 1). The cantilever beam is excited horizontally perpendicular to the beam using an electromagnetic system. A controllable current is generated and flows through the coils. The

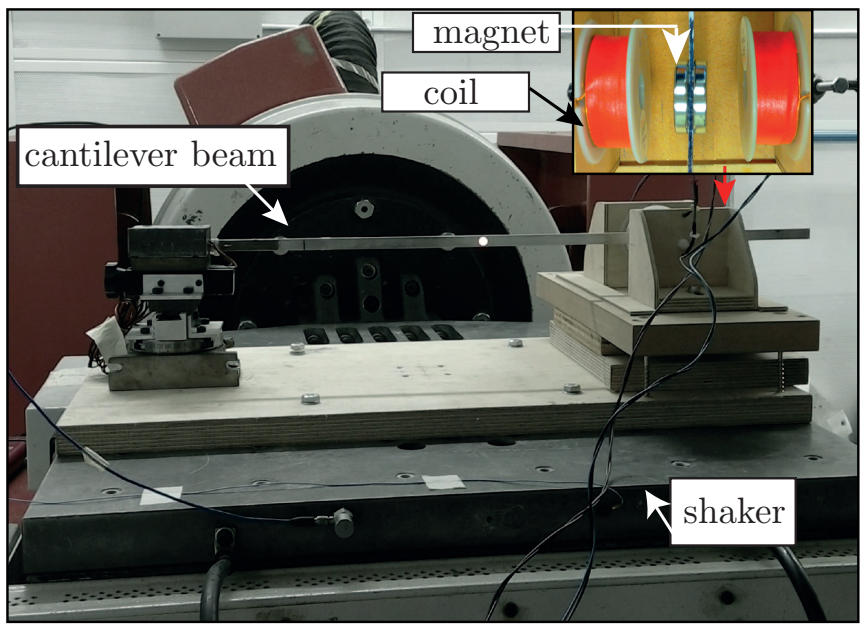

Figure 1. Experimental set-up consisting of a cantilever beam on a shaker and an electromagnetic system.

Table 1. Mechanical properties and dimensions.

\begin{tabular}{|c|c|c|}
\hline Property & Value & Units \\
\hline Radius of the magnets & 0.015 & $\mathrm{~m}$ \\
\hline Residual magnetic flux density $\left(B_{\mathrm{r}}\right)$ & 1.1 & $\mathrm{~T}$ \\
\hline Permeability $\left(\mu_{0}\right)$ & $4 \pi 10^{-7}$ & $\mathrm{NA}^{-2}$ \\
\hline Inner radius of the coil $\left(r_{1}\right)$ & 0.0085 & $\mathrm{~m}$ \\
\hline Outer radius of the coil $\left(r_{2}\right)$ & 0.0225 & $\mathrm{~m}$ \\
\hline Mean radius of the coil $\left(r_{\mathrm{c}}\right)$ & 0.0135 & $\mathrm{~m}$ \\
\hline Number of turns of in coil $(\mathrm{N})$ & 485 & - \\
\hline Length of wire in one rotation $\left(l_{\mathrm{w}}\right)$ & 0.078 & $\mathrm{~m}$ \\
\hline Diameter of the coil $\left(D_{\mathrm{w}}\right)$ & 0.00071 & $\mathrm{~m}$ \\
\hline Height of the coil with shield ( $\left.h_{\text {coil }}\right)$ & 0.02 & $\mathrm{~m}$ \\
\hline Coordinate for coil $\left(z_{1}\right)$ & 0.007 & $\mathrm{~m}$ \\
\hline Coordinate for coil $\left(z_{2}\right)$ & -0.007 & $\mathrm{~m}$ \\
\hline Resistance of the coil $\left(R_{\text {coil }}\right)$ & 1.91 & Ohm \\
\hline Inductance of the coil $\left(L_{\text {coil }}\right)$ & 0.64 & $\mathrm{mH}$ \\
\hline Load resistor $(R)$ & 0.1 & Ohm \\
\hline Width of the beam $\left(b_{\mathrm{b}}\right)$ & 0.01 & $\mathrm{~m}$ \\
\hline Thickness of the beam $\left(t_{\mathrm{b}}\right)$ & 0.002 & $\mathrm{~m}$ \\
\hline Effective mass $\left(m_{\mathrm{t}}\right)$ & 0.104 & $\mathrm{~kg}$ \\
\hline $\begin{array}{l}\text { Half of the distance between } \\
\text { the coils }(h)\end{array}$ & 0.03 & $\mathrm{~m}$ \\
\hline $\begin{array}{l}\text { Static stiffness of the beam } \\
\text { with magnets } \\
\text { and coils when } I_{\mathrm{c}}=0\left(k_{\mathrm{b}}\right)\end{array}$ & 32.84 & $\mathrm{Nm}^{-1}$ \\
\hline $\begin{array}{l}\text { Measured first natural frequency } \\
\text { of the beam with magnets and coils } \\
\text { when } I_{c}=0\left(\omega_{n, \exp }\right)\end{array}$ & 17.76 & $\operatorname{rads}^{-1}$ \\
\hline $\begin{array}{l}\text { Measured second natural frequency } \\
\text { of the beam with magnets and } \\
\text { coils when } I_{\mathrm{c}}=0\end{array}$ & 202 & $\operatorname{rads}^{-1}$ \\
\hline Mechanical damping coefficient & 0.011 & $\mathrm{Nsm}^{-1}$ \\
\hline
\end{tabular}

of the beam with magnets and

coils when $I_{\mathrm{c}}=0\left(c_{\mathrm{m}}\right)$

mechanical properties, dimensions of the cantilever beam, and the electromagnetic system are shown in Table 1. A Single Degree of Freedom (SDOF) model of a NPE system subject to base excitation is presented in Figure 2(a). In this figure, where $x$ is the displacement of moving mass $m_{\mathrm{t}}$ (the effective mass of the beam with attached magnets), $y$ is the base displacement, and $Y_{0}$ is the amplitude of harmonic 
(a)

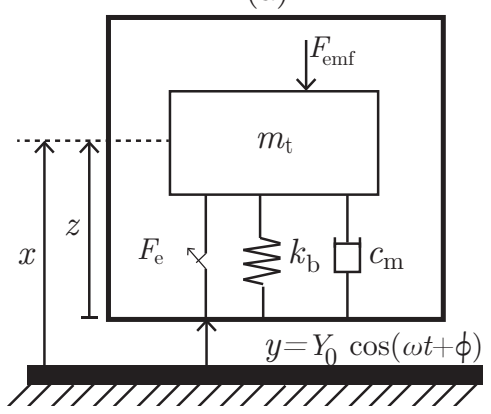

(b)

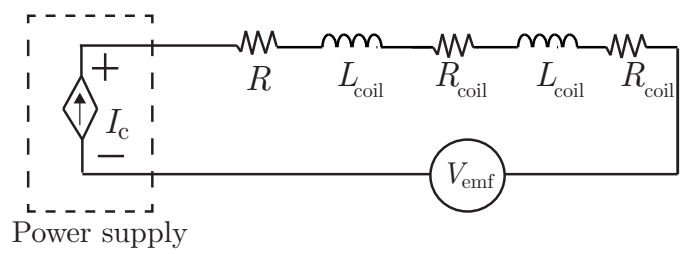

Figure 2. (a) Schematic of the base excited NPE oscillator with an electromagnetic system. (b) Circuit diagram, $L_{\text {coil }}$ is the inductance of one of the coils, $R_{\text {coil }}$ is the electrical resistance of one of the coils, $R$ is a resistor connected in series with the coils, and $V_{\mathrm{emf}}$ is the induced voltage.

base displacement at frequency $\omega$ and phase $\phi$. The relative displacement $z$ is the displacement between the mass and the base. The current $I_{\mathrm{C}}$ supplied to the coils is,

$$
I_{\mathrm{c}}=I_{\mathrm{DC}}+I_{\mathrm{AC}} \cos (\Omega t),
$$

where $I_{\mathrm{DC}}$ is the direct current and $\Omega$ is the frequency of the alternating current $I_{\mathrm{AC}}$.

The equations governing the motion of the mechanical system and the electric circuit are,

$$
m_{\mathrm{t}} \ddot{z}+c_{\mathrm{m}} \dot{z}+k_{\mathrm{b}} z+F_{\mathrm{emf}}+F_{\mathrm{e}}=m_{\mathrm{t}} \omega^{2} Y_{0} \cos (\omega t+\phi),
$$

where the overdot represents a derivative with respect to time $t, c_{\mathrm{m}}$ is the mechanical damping, $k_{\mathrm{b}}$ is the static stiffness, $F_{\text {emf }}$ is the electromagnetic force induced by the interaction of oscillating magnet and coils

$$
F_{\mathrm{emf}}=k_{\mathrm{t}}(z) i_{\mathrm{es}},
$$

where $k_{\mathrm{t}}(z)$ is electromechanical coupling factor (see Appendix A). $i_{\mathrm{es}}$ is the induced current generated by electromagnets.

$F_{\mathrm{e}}$ in equation (2) is the force applied by the electromagnetic system

$$
F_{\mathrm{e}}=H_{1}\left(I_{\mathrm{c}}+i_{\mathrm{es}}\right) z+H_{2}\left(I_{\mathrm{c}}+i_{\mathrm{es}}\right) z^{3}+\mathcal{O}\left(z^{5}\right) .
$$

where $H_{1}$ and $H_{2}$ are function of coil parameters and they are defined in Appendix A (equations (A.7) and (A.7)). Substituting equations (3) and (4) into equation (2) results in

$$
\begin{gathered}
\ddot{z}+2 \varepsilon \omega_{n}\left(\zeta_{\mathrm{m}}+\zeta_{\mathrm{es}, \mathrm{app}} z^{2}\right) \dot{z}+\omega_{n}^{2}(1+\varepsilon \delta \cos (\Omega t)) z+ \\
\omega_{n}^{2}(\varepsilon \alpha+\varepsilon \gamma \cos (\Omega t)) z^{3}=Y_{0} \omega^{2} \cos (\omega t+\phi) .
\end{gathered}
$$

In this study the base excitation frequency is considered to be half the parametric frequency $\left(\omega=\frac{\Omega}{2}\right)$, hence maximum response amplitude can be achieved when the parametric frequency is at twice the natural frequency (Zaghari et al. (2016)). Equation (5) is normalised by the time scaling $\tau=\Omega t$, and is expressed as derivatives with respect to $\tau$. Prime $(.)^{\prime}$ represents a quantity differentiated with respect to $\tau$. Normalisation in this way results in

$$
\begin{aligned}
z^{\prime \prime}+\frac{2 \varepsilon \omega_{n}\left(\zeta_{\mathrm{m}}+\zeta_{\mathrm{es}, \mathrm{app}} z^{2}\right)}{\Omega} z^{\prime} & +\frac{\omega_{n}^{2}}{\Omega^{2}}(1+\varepsilon \delta \cos (\tau)) z+ \\
\frac{\omega_{n}^{2}}{\Omega^{2}}(\varepsilon \alpha+\varepsilon \gamma \cos (\tau)) z^{3} & =\frac{1}{4} Y_{0} \cos \left(\frac{\tau}{2}+\phi\right)
\end{aligned}
$$

where $\zeta_{m}$ is the mechanical damping ratio, and $\zeta_{\text {es,app }}$ is the electrical damping ratio, which is obtained from equation (B.2). Parametric stiffness $\delta$, cubic stiffness $\alpha$, and cubic parametric stiffness $\gamma$, are defined in equations (B.3), (B.4), and (B.5). $\varepsilon$ is the small parameter.

The method of averaging is used to solve equation (6). To capture the system's near-resonance behaviour, the parametric frequency $\Omega$ varies around the reference frequency $\Omega_{0}$; the detuning parameter $\Delta$ is introduced in $\Omega=\Omega_{0}(1-\varepsilon \Delta)$ (Murdock (1999)). When the system is simplified to an undamped oscillator, the solutions of equation (6) take the form

$$
z(\tau)=a \cos \left(\frac{\omega_{n}}{\Omega_{0}} \tau-\varphi\right),
$$

where $a$ and $\varphi$ are the amplitude and the phase. The steadystate values are found from

$$
\begin{aligned}
& a^{\prime}(\tau)=-\zeta_{\mathrm{m}} a \frac{1}{\Omega}-\frac{1}{2} \delta a \frac{\omega_{n}^{2}}{\Omega^{2}} \sin (2 \varphi)-\frac{1}{4} \gamma a^{3} \frac{\omega_{n}^{2}}{\Omega^{2}} \sin (2 \varphi) \\
&+\frac{1}{4} Y_{0} \sin (\phi+\varphi)-\frac{\zeta_{\mathrm{es}, \mathrm{app}} \omega_{n}}{4 \Omega} a^{2}+\mathcal{O}\left(\varepsilon^{2}\right), \\
& a \varphi^{\prime}(\tau)=-\frac{1}{2} \Delta a-\frac{1}{2} \delta a \frac{\omega_{n}^{2}}{\Omega^{2}} \cos (2 \varphi)-\frac{3}{4} \alpha a^{3} \frac{\omega_{n}^{2}}{\Omega^{2}} \\
&-\frac{1}{2} \gamma a^{3} \frac{\omega_{n}^{2}}{\Omega^{2}} \cos (2 \varphi)+\frac{1}{4} Y_{0} \cos (\phi+\varphi)+\mathcal{O}\left(\varepsilon^{2}\right),
\end{aligned}
$$

when $a^{\prime}(\tau)=\varphi^{\prime}(\tau)=0$. Hence the amplitude is

$$
a=\frac{\Omega^{2} Y_{0} \sqrt{d_{1}^{2}+\left(p_{2}-p_{3} \cos (2 \phi)\right)^{2}}}{8 \omega_{n}^{2}\left(d_{2}-\zeta_{\mathrm{m}}^{2}-\left(\frac{1}{8} \zeta_{\mathrm{es}, \mathrm{app}} a^{2}\right)^{2}\right)},
$$

where $\quad d_{1}=-p_{1} \sin (2 \phi)+\zeta_{\mathrm{m}}+\frac{1}{8} \zeta_{\mathrm{es}, \mathrm{app}} a^{2}-p_{4} \sin (\phi)$, $d_{2}=p_{1}^{2}\left(1-\cos (2 \phi)^{2}+p_{3}^{2} \cos (2 \phi)^{2}-p_{2}^{2}\right.$,

$p_{1}=\frac{1}{4}\left(\delta+\frac{1}{2} \gamma a^{2}\right), \quad p_{2}=\frac{3}{8} \alpha a^{2}-\frac{\Omega}{2 \omega_{n}}+1, \quad$ and $p_{3}=\frac{1}{4}\left(\delta+\gamma a^{2}\right)$. Equation (6) has five stable and unstable solutions when the system parameters are non-zero $(\alpha, \delta, \gamma \neq 0)$. The effect of system parameters, such as cubic stiffness $\alpha$, parametric stiffness $\delta$, and the cubic parametric stiffness $\gamma$, and phase difference $\phi$ on response amplitude is investigated in the following sections.

\section{Nonlinear system with positive cubic stiffness}

A nonlinear system has positive cubic stiffness when $\alpha>0$ and $\delta=\gamma=0$ in equation (6). The effect of cubic stiffness $\alpha$ 


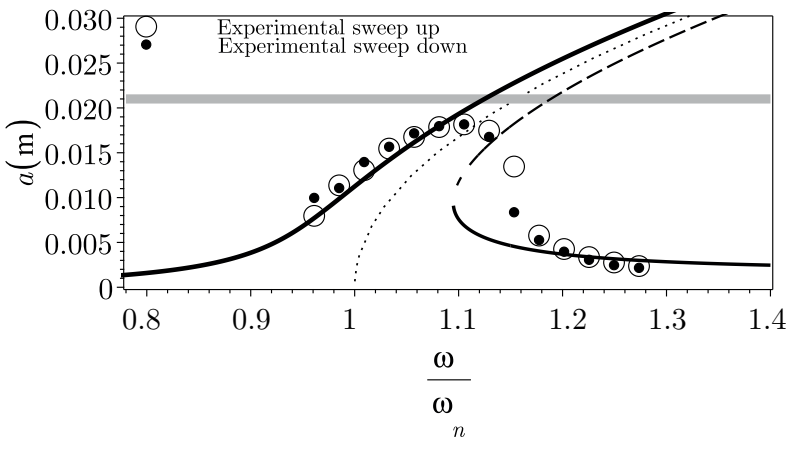

Figure 3. Analytical and experimental amplitude-frequency relation, $a$ versus $\frac{\omega}{\omega}$. The dotted line is the backbone curve. The solid lines are the stable branches, and the dashed line is the unstable branch. The grey bar shows the limit for the beam transverse vibration above which the magnets hit the coils. The coil distance $h$ is chosen to be equal to $0.03 \mathrm{~m}$, hence the maximum displacement of the beam is $0.022 \mathrm{~m}$.

on the response is investigated experimentally in this section, and these results are compared with expected behaviour from the analytical model. Figure 3 shows the analytical and experimental amplitude-frequency relation. Analytical results show that the peak amplitude is shifted to a frequency higher than resonance frequency due to the hardening nonlinearity.

The experiment is set up to achieve zero cubic parametric stiffness $\gamma$, so that the effect of the hardening nonlinearity is studied independently. This is obtained by setting $I_{\mathrm{AC}}=0$ when $I_{\mathrm{DC}}=0.38 \mathrm{~A}$. The cantilever beam is placed on the shaker and excited with the displacement amplitude $Y_{0}=0.001 \mathrm{~m}$. This small displacement amplitude is considered to prevent the magnets from hitting the coils. Equation (B.4) shows that the cubic stiffness is $\alpha=937.58 \mathrm{~m}^{-2}$, and equation (B.2) shows that the nonlinear damping is $\zeta_{\mathrm{es}, \text { app }}=217.8$. The linear natural frequency and the linear mechanical damping ratio are measured from impact tests as $\omega_{\mathrm{n}}=32.59 \mathrm{rad} \mathrm{s}^{-1}$ and $\zeta_{\mathrm{m}}=0.001$, respectively. More information regarding these measurements can be found in Zaghari (2016).

Figure 3 shows the amplitude-frequency relation for step up and down tests, which are carried out by increasing and decreasing the base excitation (shaker) frequency. For the step up test, the shaker frequency increases from $5 \mathrm{~Hz}$ to $8 \mathrm{~Hz}$ at a rate of $0.0025 \mathrm{~Hz} / \mathrm{sec}$, with constant displacement amplitude $Y_{0}=0.001 \mathrm{~m}$ at all frequencies. Each experimental point in Figure 3 corresponds to the amplitude of the steady-state response (the amplitude of the relative displacement $z(t))$ at some selected frequencies. The beam displacement $x(t)$ is subtracted by the base displacement $y(t)$ to find the relative displacement. The cantilever beam velocity is measured by the vibrometer, and is integrated numerically with respect to time to find the beam displacement. The acceleration of the shaker is recorded by an accelerometer attached to the shaker plate, which is integrated with respect to time twice to find the displacement of the shaker $y(t)$. The velocity response and the acceleration signal are filtered with a fourth-order Butterworth high pass filter with a cutoff frequency of $1 \mathrm{~Hz}$.
The experimental results show that the hardening nonlinearity shifts the maximum peak amplitude to a higher frequency. When the response amplitude is large $(a>0.017 \mathrm{~m})$ and the magnets are close to the coils, there are discrepancies between the experimental and analytical results. This is due to the varying inclination of the magnets with the beam at large amplitudes (Liu and Liu (2006)), which is not considered in our mathematical model. For example, between $\frac{\omega}{\omega_{n}}=1.1$ and $\frac{\omega}{\omega_{n}}=1.15$, there are experimental points close to the unstable branch. In this frequency region the stable steadystate solutions from the analytical stable branch do not match with the steady-state solutions from experiments. In other frequency regions where the magnets are not close to the coils, the experimental and analytical results are in good agreement.

\section{The effects of parametric stiffness for varying phase}

The effect of parametric stiffness $\delta$ and phase difference $\phi$ on the response amplitude is investigated in this section. Owing to the difficulty in controlling the phase difference between the current and the base excitation, the frequency sweep method used generally in nonlinear systems to find the stable branches has not been applied in this investigation. Instead, each frequency is considered independently in the experimental analysis. This investigation requires the phase difference between the base and parametric excitation $\phi$ to be varied; $\phi$ is controlled by adjusting the phase between the shaker acceleration and current supplied to the coils. To generate a current with various phase differences with the acceleration of the shaker, a separate hardware was programmed to generate current with different phase. More details are explained in Zaghari (2016).

Figure 4 shows the stable solutions versus phase $\phi$ when parametric stiffness is below the instability threshold $\left(\delta<\delta_{\mathrm{th}}\right)$, and when it is above the instability threshold $\left(\delta>\delta_{\mathrm{th}}\right)$ where $\delta_{\mathrm{th}}=4 \zeta_{\mathrm{m}}$. The mathematical model shows that increasing the parametric stiffness over the instability threshold increases the response amplitude for all phases on both the stable branch (shown by the black lines in Figure 4) and on the additional branch shown by the grey line. Figure 4(a) shows that the additional branch does not exist when the parametric stiffness in under the instability threshold. This is due to the influence of parametric resonance on the response of the NPE system. The experimental and analytical results in Figure 4 show that the minimum and maximum response amplitudes occur at phases $\phi=0 \mathrm{rad}$ and $\phi= \pm \frac{\pi}{2} \mathrm{rad}$, respectively. Figure 4 also shows that the phase that maximises or minimises the response amplitude does not change for varying $\delta$ and $\gamma$. This behaviour is different from the system presented in Kumar et al. (2011), because $\delta$ is coupled to $\alpha$ in that system. Figures 4(b) and 4(c) show that, during an experiment, the motion dynamics can match either the stable branch, or the additional stable branch. This cannot be easily controlled because the response can jump from one branch to the other with little perturbation, particularly when the solutions have a similar response amplitude. 
(a)

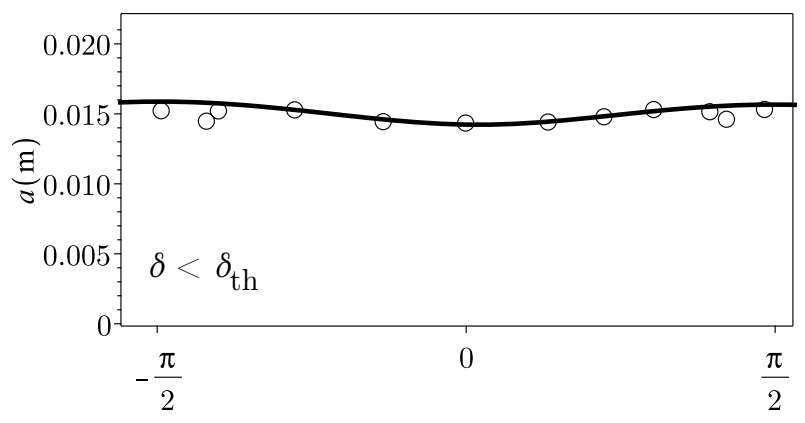

$\phi$

(b)

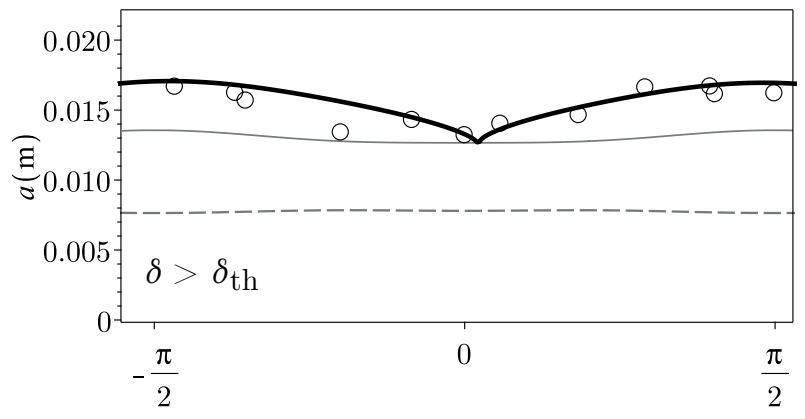

$\phi$

(c)

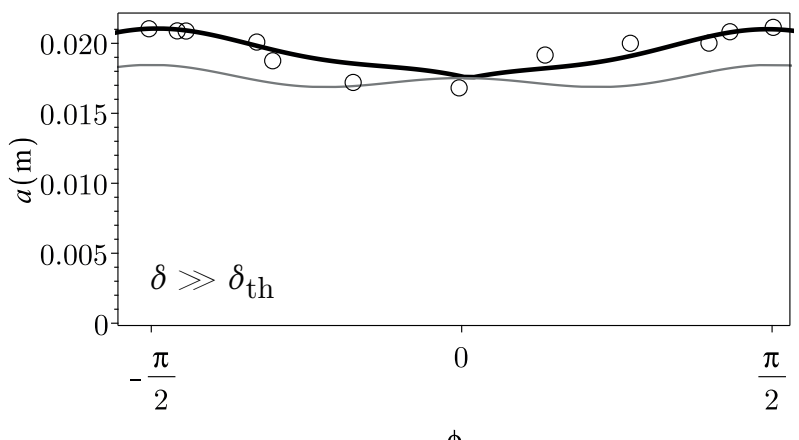

$\phi$

Figure 4. Amplitude of the steady-state response versus phase $\phi$ for the NPE system (a) below the instability threshold $(\delta=0.0027)$, (b) slightly above the instability threshold $(\delta=0.068)$, and (c) above the instability threshold $(\delta=0.218)$. The system parameters are given in Table 2 . The thick black lines represent stable solutions caused by base and parametric excitation, the thin grey line represents additional stable solutions caused by only the parametric excitation, and the dashed line represent the unstable branch. Points shown by $\circ$ denote experimental results.

\section{The effects of parametric frequency for varying phase}

In this section, the effects of parametric frequency $\Omega$ and phase difference $\phi$ on the response amplitude are determined. The amplitude-frequency relations are plotted analytically in Figure 5 for $Y_{0}=0.001 \mathrm{~m}, \zeta_{\mathrm{m}}=0.001$, $\omega_{\mathrm{n}}=36.8 \mathrm{rad} \mathrm{s}^{-1}$, and for phase differences $\phi=\frac{\pi}{2} \mathrm{rad}$ and $\phi=0 \mathrm{rad}$. $\zeta_{\text {es }, \text { app }}=142.58, \delta=0.167, \alpha=1031 \mathrm{~m}^{-2}$, and $\gamma=238.12 \mathrm{~m}^{-2}$ are calculated from equations (B.2) - (B.5) for $I_{\mathrm{DC}}=0.52 \mathrm{~A}$ and $I_{\mathrm{AC}}=0.1 \mathrm{~A}$. The analytical results show that the amplitude of the response is minimised on
Table 2. System parameters

\begin{tabular}{lccc}
\hline Parameters & Figure 4(a) & Figure 4(b) & Figure 4(c) \\
\hline \multicolumn{4}{c}{ Measured parameters } \\
\hline$I_{\mathrm{DC}}(\mathrm{A})$ & 0.54 & 0.54 & 0.54 \\
$I_{\mathrm{AC}}(\mathrm{A})$ & 0.002 & 0.05 & 0.16 \\
$Y_{0}(\mathrm{~m})$ & 0.001 & 0.001 & 0.001 \\
$\omega_{\mathrm{n}}\left(\mathrm{rad} \mathrm{s}^{-1}\right)$ & 37.3 & 37.3 & 37.3 \\
$\Omega\left(\mathrm{rad} \mathrm{s}^{-1}\right)$ & $2 \omega$ & $2 \omega$ & $2 \omega$ \\
$\omega\left(\mathrm{rad} \mathrm{s}^{-1}\right)$ & $\omega_{\mathrm{n}}$ & $\omega_{\mathrm{n}}$ & $\omega_{\mathrm{n}}$ \\
$\zeta_{\mathrm{m}}$ & 0.001 & 0.001 & 0.001 \\
\hline \multicolumn{3}{c}{ Calculated parameters from equations (B.2) $^{-(B .5)}$} \\
\hline$\zeta_{\mathrm{es}, \text { app }}$ & 144.51 & 144.51 & 144.51 \\
$\delta$ & 0.0027 & 0.068 & 0.218 \\
$\alpha\left(\mathrm{m}^{-2}\right)$ & 1042.40 & 1042.40 & 1042.40 \\
$\gamma\left(\mathrm{m}^{-2}\right)$ & 3.86 & 96.52 & 308.86
\end{tabular}

the upper stable branch when $\phi=0$, and is maximised when $\phi=\frac{\pi}{2}$. The opposite is true for the lower stable branch.

Five distinct response branches are shown in Figure 5. The two additional branches relative to Figure 3 arise from the additional resonance frequencies in the nonlinear parametric system. One resonance frequency is induced by a combination of base and parametric excitation, and the other is caused by only the parametric excitation. Previous studies assume that, for a NPE system without nonlinear damping, a change in the phase difference does not affect additional branches (grey lines in Figure 5). However this study shows that, since the parametric frequency changes the response amplitude, the change in the response amplitude affects the nonlinear damping, which causes the additional branch to change.

Labels (a)-(f) in Figure 5 indicate individual experiments conducted at different parametric frequencies $\Omega$. The amplitude-frequency relation plots at each frequency are shown in Figure 6. The experimental results in Figure 6 agree with the mathematical model, in that the response amplitude of the NPE system with $\phi=\frac{\pi}{2} \mathrm{rad}$ is greater than the response of the nonlinear system without parametric stiffness shown in Figure 3. The experimental results presented in Figure 6 also show that the response amplitude is minimised on the upper stable branch when $\phi=0$, and is maximised when $\phi=\frac{\pi}{2}$ (Figure 6(a-e)), while the opposite is true for the lower branch (Figure 6(f)). Between labels (e)-(f) in Figure 5, where $2.15 \leq \frac{\Omega}{\omega_{n}} \leq 2.62$, no experimental data was gathered because the response amplitude caused the magnets to hit the coils. Also, when the response amplitude is large and the magnets are close to the coils, there are discrepancies between the experimental and analytical results due to the inclination of the magnets.

\section{Conclusion}

This paper introduces a base-excited cantilever beam with an electromagnetic subsystem as a prototypical NPE system. This system allows cubic stiffness, parametric stiffness, cubic parametric stiffness, and the phase difference between the base excitation and the excitation from the electromagnetic subsystem to be varied independently, so their individual effect on the amplitude of the response can be determined. A mathematical model of this physical 
(a)

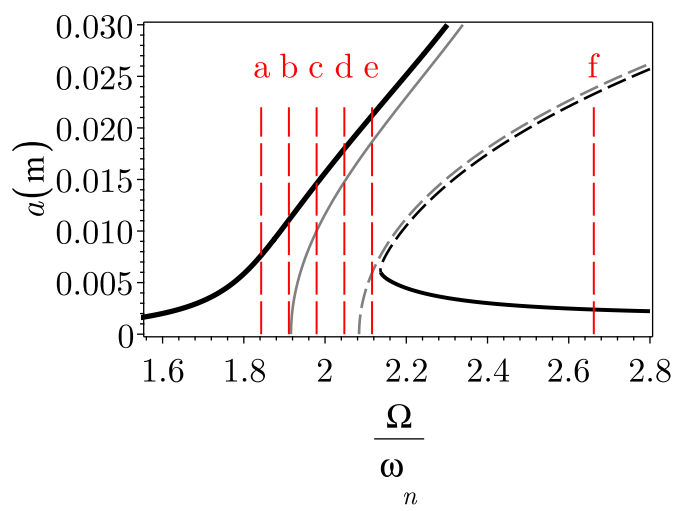

(b)

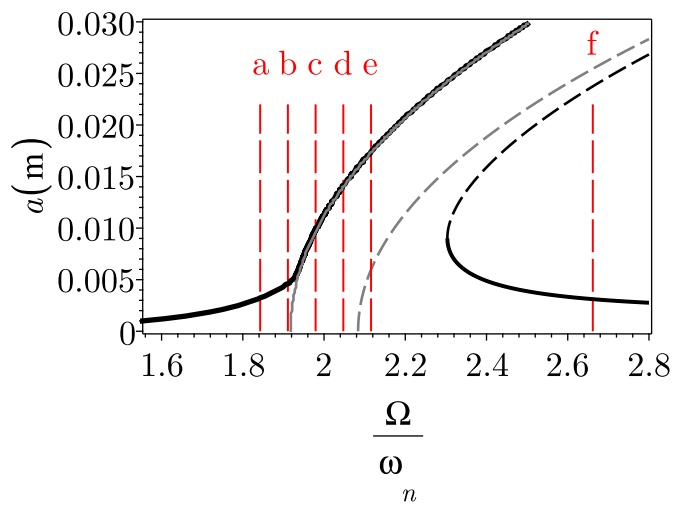

Figure 5. Analytical amplitude-frequency relations for (a) $\phi=\frac{\pi}{2}$, and (b) $\phi=0$. Solid lines denote stable branches, and dashed lines denote unstable branches. Grey lines denote additional branches. Labels (a)-(f) mark individual experiments conducted at different parametric frequencies $\Omega$. The values of these experiments are mapped onto the amplitude-frequency relation plots in Figure 6.

system is also presented to demonstrate general NPE system behaviour; good agreement is found between the mathematical model and the experiments.

By increasing cubic stiffness, and consequently hardening nonlinearity, the response frequency of the peak response amplitude was increased. This agrees with studies conducted in the literature (Neumeyer et al. (2016)), demonstrating compatibility of this system with existing models.

An increase in parametric stiffness increases the response amplitude, though the magnitude of this increases depends strongly on the phase difference between the excitation and the response. On the upper-stable branch, the response amplitude is maximised at phase $\phi=\frac{\pi}{2}$. However, the response on the lower-stable branch is maximised at $\phi=0$. This result holds regardless of the parametric stiffness and the cubic parametric stiffness. This phase relationship significantly impacts the design of energy harvesters and amplifiers, where a greater response amplitude is desirable, and where the branch of the response and the phase difference can be both controlled.

\section{References}

Chen L, He F and Sammut K (2009) Vibration suppression of a principal parametric resonance. Journal of Vibration and (a)

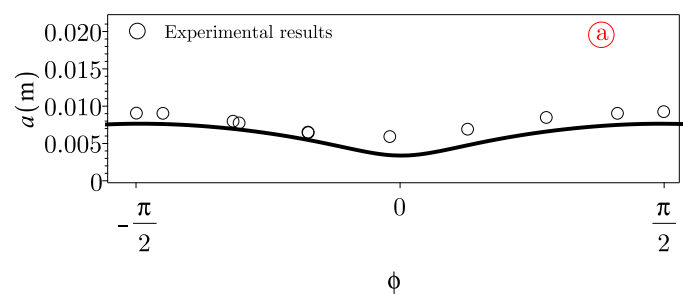

(b)

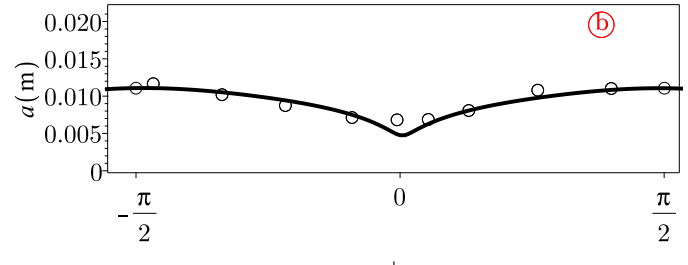

(c)

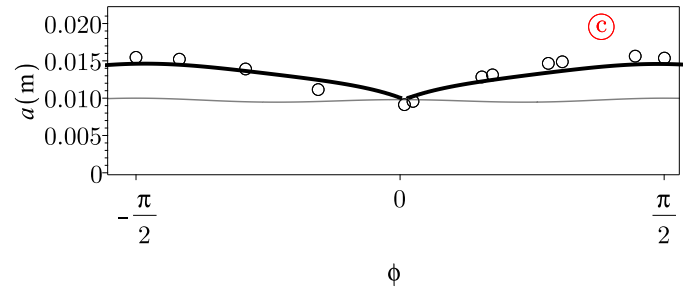

(d)

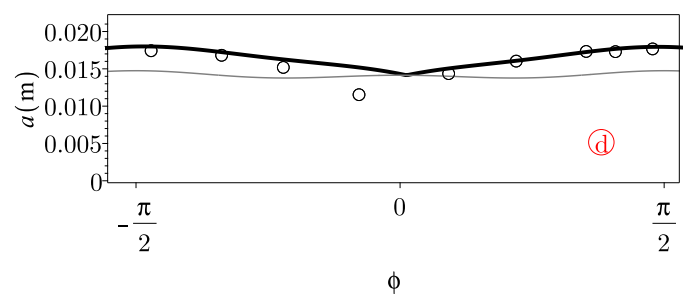

(e)

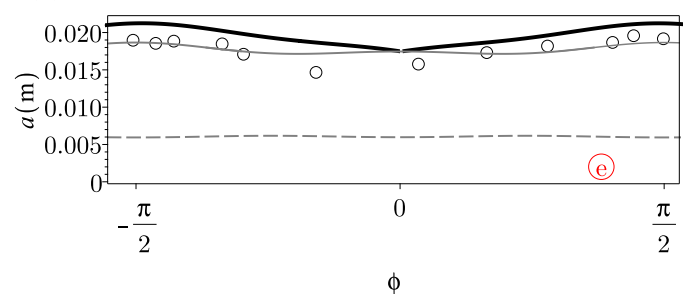

(f)

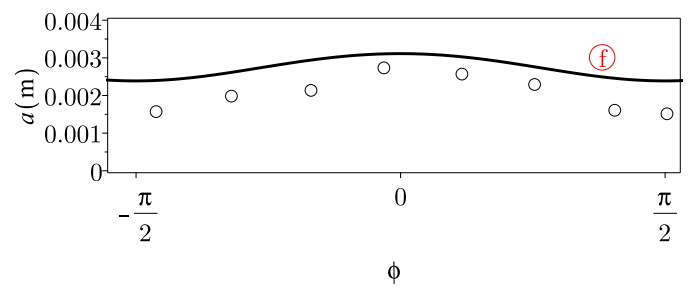

Figure 6. Analytical and experimental results for labels (a)-(f) denote in Figure 5. Solid lines denote stable branches, and dashed lines denote unstable branches. Grey lines denote additional branches.

Control 15(3): 439-463.

DeMartini B, Moehlis J, Turner K, Rhoads J, Shaw S and Zhang W (2005) Modeling of parametrically excited microelectromechanical oscillator dynamics with application 
to filtering. In: Proceedings of the Fourth IEEE Conference on Sensors. IEEE, pp. 345-348.

Hammond P (2013) Electromagnetism for engineers: an introductory course. Elsevier.

Hu ZX, Gallacher BJ, Burdess JS, Fell CP and Townsend K (2011) A parametrically amplified mems rate gyroscope. Sensors and Actuators A: Physical 167(2): 249-260.

Kumar V, Miller JK and Rhoads JF (2011) Nonlinear parametric amplification and attenuation in a base-excited cantilever beam. Journal of Sound and Vibration 330(22): 5401-5409.

Liu J and Liu K (2006) A tunable electromagnetic vibration absorber: characterization and application. Journal of Sound and Vibration 295(3): 708-724.

Murdock JA (1999) Perturbations: theory and methods, volume 27. Siam.

Nayfeh AH and Mook DT (2008) Nonlinear oscillations. John Wiley \& Sons.

Neumeyer S, Sorokin VS and Thomsen JJ (2016) Effects of quadratic and cubic nonlinearities on a perfectly tuned parametric amplifier. Journal of Sound and Vibration 386: 327 335.

Rhoads JF, Miller NJ, Shaw S and Feeny B (2008) Mechanical domain parametric amplification. Journal of Vibration and Acoustics 130(6): 061006.

Rhoads JF and Shaw SW (2010) The impact of nonlinearity on degenerate parametric amplifiers. Applied Physics Letters 96(23): 234101

Sneller AJ and Mann BP (2010) On the nonlinear electromagnetic coupling between a coil and an oscillating magnet. Journal of Physics D: Applied Physics 43(29): 295005.

Tusset AM, Janzen FC, Piccirillo V, Rocha RT, Balthazar JM and Litak G (2017) On nonlinear dynamics of a parametrically excited pendulum using both active control and passive rotational (MR) damper. Journal of Vibration and Control 24(9): 1587-1599.

Zaghari B (2016) Dynamic analysis of a nonlinear parametrically excited system using electromagnets. $\mathrm{PhD}$ Thesis, Institute of Sound and Vibration Research (ISVR), University of Southampton, UK.

Zaghari B, Ghandchi Tehrani M and Rustighi E (2014) Mechanical modelling of a vibration energy harvester with time-varying stiffness. In: EURODYN 2014: 9th International Conference on Structural Dynamics. pp. 2079-2086.

Zaghari B, Rustighi E and Ghandchi Tehrani M (2016) Dynamic response of a nonlinear parametrically excited system subject to harmonic base excitation. In: Journal of Physics: Conference Series, volume 744. IOP Publishing, p. 012125.

\section{Appendix A}

\section{Mathematical model of the electromagnetic system}

Based on Lenz's law, the force $F_{\text {emf }}$ in equation (2) is proportional to the induced current in coils $i_{\mathrm{es}}$,

$$
F_{\mathrm{emf}}=k_{\mathrm{t}}(z) i_{\mathrm{es}},
$$

where $k_{\mathrm{t}}(z)$ is electromechanical coupling factor.
When the magnet is moved along the axis of the coil, an electric potential across the coils is generated.

$$
V_{\mathrm{emf}}=\frac{\mathrm{d}}{\mathrm{d} t} \int_{A} \mathbf{B} \mathrm{d} A=k_{\mathrm{t}}(z) \dot{z},
$$

where $\mathbf{B}$ is the magnetic field generated by the moving magnet, $A$ indicates the area enclosed by the wire loop, $z$ is the magnet displacement. The quantity $k_{\mathrm{t}}$ for two coils in series and a magnet, based on (Sneller and Mann (2010)) is

$$
k_{\mathrm{t}}(z)=\frac{3 \varsigma V_{\mathrm{m}} B_{\mathrm{r}}}{2\left(r_{2}-r_{1}\right)\left(z_{2}-z_{1}\right)}\left(\sum_{n, m=1}^{2}(-1)^{n+m} z_{\mathrm{nm}}\right),
$$

$$
\begin{gathered}
z_{\mathrm{nm}}=\ln \left(r_{\mathrm{n}}+z_{\mathrm{nm}}^{\prime}\right)-\frac{r_{\mathrm{n}}}{z_{\mathrm{nm}}^{\prime}}-\ln \left(r_{\mathrm{n}}+z_{\mathrm{nm}}^{\prime \prime}\right)+\frac{r_{\mathrm{n}}}{z_{\mathrm{nm}}^{\prime \prime}} \\
z_{\mathrm{nm}}^{\prime}=\sqrt{r_{\mathrm{n}}^{2}+\left(z_{\mathrm{m}}-(z+h)\right)^{2}} \\
z_{\mathrm{nm}}^{\prime \prime}=\sqrt{r_{\mathrm{n}}^{2}+\left(z_{\mathrm{m}}-(-z+h)\right)^{2}}
\end{gathered}
$$

where the fill factor $\varsigma$ is

$$
\varsigma=\frac{D_{\mathrm{w}}^{2} l_{\mathrm{w}} N}{4\left(r_{2}^{2}-r_{1}^{2}\right) h_{\text {coil }}},
$$

$V_{\mathrm{m}}$ is the volume of the magnet, $B_{\mathrm{r}}$ is the residual magnetic flux density, $D_{\mathrm{w}}$ is the coil diameter, $l_{\mathrm{w}}$ is the length of the wire in one rotation, $N$ is the number of turns in each coil, $h_{\text {coil }}$ is the height of the coil, $z_{1}$ and $z_{2}$ refer to the height of the coil, and $r_{1}$ and $r_{2}$ are the inner and outer radius of the coil respectively. Note that assumptions for the nonlinear coupling coefficient may not be valid for all coil configurations, and careful consideration is needed when the electromagnetic system geometry is varied.

In equation 2 the electromagnetic force $F_{\mathrm{e}}=-\mu \cdot \frac{\mathrm{dB}_{\mathrm{z}}}{d z} \quad$ (Hammond (2013)), where $\mu$ is the magnitude of magnetic moment and the magnetic field $B_{\mathrm{z}}$ generated by the two pairs of coils can be obtained from the Biot-Savart law,

$$
B_{\mathrm{z}}(z)=-\frac{\mu_{0} r_{\mathrm{c}}^{2}\left(I_{\mathrm{c}}+i_{\mathrm{es}}\right) N}{2\left((h+z)^{2}+r_{\mathrm{c}}^{2}\right)^{\frac{3}{2}}}-\frac{\mu_{0} r_{\mathrm{c}}^{2}\left(I_{\mathrm{c}}+i_{\mathrm{es}}\right) N}{2\left((z-h)^{2}+r_{\mathrm{c}}^{2}\right)^{\frac{3}{2}}},
$$

where $h$ is the distance between the centre of coil and the equilibrium position, and other parameters are defined in Table 1. Using the Taylor expansion about $z=0$, the force applied to the cantilever beam can be expressed as

$$
F_{\mathrm{e}}=H_{1}\left(I_{\mathrm{c}}+i_{\mathrm{es}}\right) z+H_{2}\left(I_{\mathrm{c}}+i_{\mathrm{es}}\right) z^{3}+\mathcal{O}\left(z^{5}\right),
$$

where $H_{1}$ and $H_{2}$ are

$$
\begin{gathered}
H_{1}=\frac{-2 G}{\left(h^{2}+r_{\mathrm{c}}^{2}\right)^{\frac{5}{2}}}+\frac{10 G h^{2}}{\left(h^{2}+r_{\mathrm{c}}^{2}\right)^{\frac{7}{2}}}, \\
H_{2}=\frac{5 G}{\left(r_{\mathrm{c}}^{2}+h^{2}\right)^{\frac{7}{2}}}-\frac{70 G h^{2}}{\left(r_{\mathrm{c}}^{2}+h^{2}\right)^{\frac{9}{2}}}+\frac{105 G h^{4}}{\left(r_{\mathrm{c}}^{2}+h^{2}\right)^{\frac{11}{2}}}, \\
G=\frac{3}{2} \mu \mu_{0} r_{c}^{2} N .
\end{gathered}
$$




\section{Appendix B}

\section{Parameter identification}

Substituting $F_{\text {emf }}$ (equation (A.1)) and $F_{\mathrm{e}}$ (equation (A.6)) into equation (6) and normalising by the total effective mass $m_{\mathrm{t}}$ results in

$$
\begin{gathered}
\ddot{z}+2 \varepsilon \omega_{n}\left(\zeta_{\mathrm{m}}+\zeta_{\mathrm{es}, \mathrm{app}} z^{2}\right) \dot{z}+\omega_{n}^{2}(1+\varepsilon \delta \cos (\Omega t)) z+ \\
\omega_{n}^{2}(\varepsilon \alpha+\varepsilon \gamma \cos (\Omega t)) z^{3}=Y_{0} \omega^{2} \cos (\omega t+\phi) .
\end{gathered}
$$

In equation (B.1) small parameter $\varepsilon$ is added to solve this equation with the method of averaging, the mechanical damping ratio $\zeta_{\mathrm{m}}=\frac{c_{\mathrm{m}}}{2 m_{\mathrm{t}} \omega_{\mathrm{n}}}$, and $\zeta_{\mathrm{es} \text {,app }}$ is the approximate electrical damping applied from the electromagnetic system. The dissipative feedback force due to the electromagnetic system can be calculated from equation (A.2). The electrical damping ratio is

$$
\zeta_{\text {es }, \text { app }}=\frac{k_{\mathrm{t}, \mathrm{app}}^{2}}{2 m_{\mathrm{t}} \omega_{\mathrm{n}}\left(2 R_{\mathrm{coil}}+R\right)},
$$

where first natural frequency of the cantilever beam with the electromagnetic system $\omega_{\mathrm{n}}$ is estimated using the Rayleigh Energy Method.

The change of electromechanical coupling with respect to $z$ is considered linear $k_{t}(z)=k_{\mathrm{t} \text {,app }} z+\mathcal{O}\left(z^{2}\right)$, where $k_{\mathrm{t}, \mathrm{app}}=\left.\frac{\partial k_{\mathrm{t}}(z)}{\partial z}\right|_{z=0}$.

In equation (6), normalised dimensionless parametric stiffness $\delta$, normalised dimensionless cubic stiffness $\alpha$, and normalised dimensionless cubic parametric stiffness $\gamma$ are derived from electromagnetic forces

$$
\delta=\frac{H_{1} I_{\mathrm{AC}}}{k_{1}},
$$

where $k_{1}=k_{\mathrm{b}}+I_{\mathrm{DC}} H_{1}$ is the total linear stiffness. The normalised cubic stiffness $\alpha$ is

$$
\alpha=\frac{H_{2} I_{\mathrm{DC}}}{k_{1}} .
$$

The cubic stiffness is strongly affected by the direct current and the parameters of the electromagnetic system, such the distance between the coils, the number of turns in each coil, and the mean radius of the coil. The normalised cubic parametric stiffness is

$$
\gamma=\frac{H_{2} I_{\mathrm{AC}}}{k_{1}}
$$

